\title{
Forum
}

\section{Sailing in Ever-Decreasing Circles}

\author{
Roger Prince and Roy Williams
}

1. INTRODUCTION. While it is clearly established that the shortest distance between two points on the surface of a sphere lies along the arc of a Great Circle, is it fecund to investigate exactly how much a Great Circle saves in distance in comparison to a Small Circle? In particular, it has been shown that, where navigation is restricted (perhaps by ice) to any particular maximum latitude, the shortest route is a Composite Great Circle, such that the approach to, and retreat from, that limiting latitude are the Great Circles for which the limiting latitude is the vertex.

The definition of a Great Circle is a planar circle the plane of which passes through the centre of the sphere. The plane of a Small Circle does not have this restriction, so that while there is only one Great Circle which passes through any two points on the surface (these two points and the centre of the sphere defining the plane in which the circle lies), there is an infinity of Small Circles which do so, cutting the sphere with everdecreasing radii, until the two points are at opposite ends of the diameter formed by a chord of the sphere.

It is a paradox that, while all Small Circles passing through those two points have necessarily smaller diameters than the corresponding Great Circle, ex hypothesi the distance between those two points along the arc of a Small Circle will be greater the smaller the radius of the Small Circle until, in the limit, that distance becomes a semicircumference.

We also find that, in the limit, the ratio of the distances between two points along the Great Circle and the Small Circle routes is the ratio of the semi-circumference of the Small Circle to its diameter, i.e. $\frac{1}{2} \pi$. To demonstrate this the extreme cases can be considered.

(i) Where the two points are at the opposite ends of a diameter of the Earth's surface (such as the poles) when all circles passing through them will be Great Circles which, in the case of the poles, are the meridians of longitude and the distances between the two points along these arcs are identical.

(ii) At the other end of the scale, where the points tend to coincidence, the distance between them along the arc of the unique Great Circle tends to the diameter of the smallest Small Circle on which they also lie. Thus the proportional increase between the distance travelled along the semi-circumference of the Small Circle and the distance travelled along the arc of the Great Circle is $\frac{1}{2} \pi-\mathrm{I}$, i.e. approximately 57 percent. This, then, is the maximum extra distance which has to be travelled by using the Small Circle route between two points rather than the path along the corresponding the Great Circle.

What then of an intermediate situation? Let us suppose that we wish to travel between the points $\left(30^{\circ} \mathrm{N}, \mathrm{O}^{\circ} \mathrm{E} / \mathrm{W}\right)$ and $\left(30^{\circ} \mathrm{N}, 180^{\circ} \mathrm{E} / \mathrm{W}\right)$. The shortest distance is obviously the Great Circle route over the North Pole and its value is $2 \pi R / 3$ where $R$ is the radius of the sphere. If, however, navigation is restricted for some reason so that there is a limiting latitude, let us consider two examples: 
(i) Where navigation is restricted to the parallel $30^{\circ} \mathrm{N}$. In this example the Small Circle route will be along this parallel. The radius of the parallel is $\frac{1}{2}(R \sqrt{ } 3)$ and the length of arc along the half circle will be $\frac{1}{2} \pi(R \sqrt{ } 3)$. The extra distance travelled, therefore, by not using the Great Circle route is $\pi R(3 \sqrt{3}-4) / 6$ and the ratio of the increase is $(3 \sqrt{3}-4) / 4$ which is approximately 30 per cent. In this example the Composite Great Circle route is identical to the small circle of the limiting latitude no part of the composite route follows the path of a Great Circle.

(ii) Where navigation is restricted to the parallel $60^{\circ} \mathrm{N}$. We have the situation which appears in the diagram shown in Fig. I where $A, B$ are the end points and $V$ is the halfway

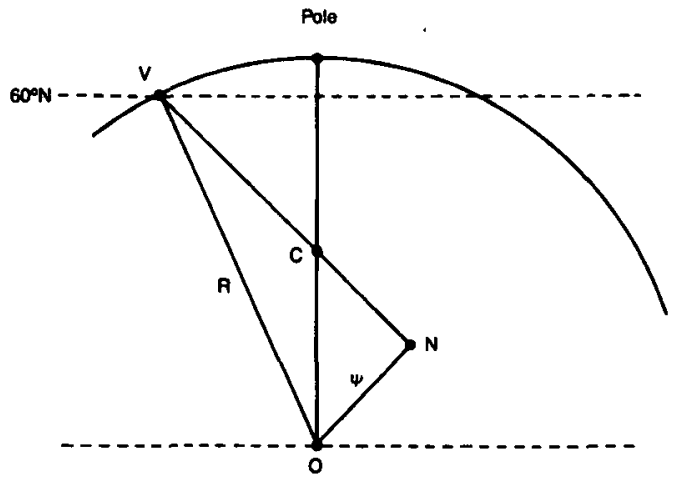

Fig. I

point (the vertex) in latitude $60^{\circ} \mathrm{N}$ at longitude $90^{\circ} \mathrm{E} / \mathrm{W}$. The figure is in the plane of the meridian through $V . N$ is the centre of the Small Circle which has its vertex in the limiting latitude.

In order to find the distance we have to travel along the Small Circle route we need to calculate the radius, $V N$, of the Small Circle and the angle subtended at the centre of the Small Circle by the points $A$ and $B$. We also need to calculate the perpendicular distance from $N$ to the chord $A B$. This distance is $C N$. The perpendicular distance from $O$ to $A B$ is then $O C$ and, since $A$ and $B$ are at the opposite ends of the diameter of a Small Circle and in the same latitude, then

$$
O C=R \sin 30^{\circ}
$$

From Fig. I we see that the distance $O N$ is

$$
O N=R \cos \left(\psi+30^{\circ}\right)
$$

and, at the same time,

$$
O N=O C \cos \psi
$$

so that, combining these results we find

$$
R \cos \left(\psi+30^{\circ}\right)=R \sin 30^{\circ} \cos \psi
$$

from which we find

$$
\tan \psi=\sqrt{ } 3-1 \text { and } \psi=36.2^{\circ}
$$

Hence the radius $V N(=r$, say) of the Small Circle is

$$
r=R \sin \left(\psi+30^{\circ}\right)=0.915 R
$$


and the distance $C N$ is given by

$$
C N=R \sin 30^{\circ} \sin \psi=0.2953 R
$$

The angles $V N A$ and $V N B$ are both equal to

$$
\cos ^{-1}(C N / r)=71 \cdot 17^{\circ}
$$

and the full angle subtended by the chord $A B$ at $N$ is

$$
142 \cdot 34^{\circ}
$$

The length of arc $A B$ along the Small Circle centred at $N$ is therefore

$$
\pi r(142.34 / 180)=2.2732 R
$$

The difference in distance between this and the distance by the Great Circle route over the pole is

$$
2 \cdot 2732 R-(2 \pi / 3) R=0.1788 R
$$

which is approximately 8.5 percent of the Great Circle distance.

The distance by the composite route is found to be $2.2505 R$ and the difference in distance between this and the Small Circle route is $0.0227 R$ which is only some 1 percent. This is the extra travelled by using the Small Circle route rather than the composite route.

2. TO DETERMINE THE GENERAL EQUATION OFA SMALL CIRCLE BETWEEN TWO GIVEN POINTS AND THE PLANE IN WHICH IT LIES. The general equation of a plane in rectangular Cartesian coordinates $x, y, z$ is given by

$$
z=a x+b y+c
$$

where $a, b, c$ are constants determined from three non-collinear points in the plane. The value of $c$ is the value of $z$ when $x=y=0$.

If we have a sphere of radius $r$ centred at the origin of coordinates and whose axis corresponds with the $z$ axis of the Cartesian frame we can transform equation ( 1 ) using the transformations

$$
\begin{aligned}
& x=r \cos \phi \cos \theta \\
& y=r \cos \phi \sin \theta \\
& z=r \sin \phi
\end{aligned}
$$

then we find that a circle in the plane is defined by

$$
\sin \phi=a \cos \phi \cos \theta+b \cos \phi \sin \theta+c
$$

Differentiating (2) we find

$$
\begin{aligned}
\cos \phi \frac{d \phi}{d \theta}= & {\left[-a \sin \phi \cos \theta \frac{d \phi}{d \theta}-a \cos \phi \sin \theta\right] } \\
& +\left[-b \sin \phi \sin \theta \frac{d \phi}{d \theta}+b \cos \phi \cos \theta\right]
\end{aligned}
$$

At the vertex (the point on the circle at which $\phi$ is maximum) we must have $d \phi / d \theta=\circ$ so that

$$
-a \cos \phi_{v} \sin \theta_{v}+b \cos \phi_{v} \sin \theta_{v}=0
$$

where $\phi_{v}$ and $\theta_{v}$ are the latitude and longitude of the vertex. 


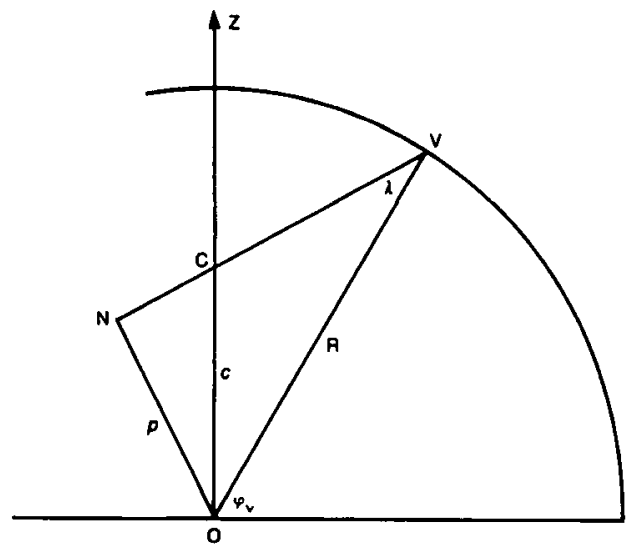

Fig. 2

Hence

$$
\tan \theta_{v}=\frac{b}{a}
$$

and

$$
\sin \theta_{v}=\frac{b}{\sqrt{ }\left[a^{2}+b^{2}\right]}, \quad \cos \theta_{v}=\frac{a}{\sqrt{ }\left[a^{2}+b^{2}\right]}
$$

Substituting these results into equation (2) gives us

$$
\sin \phi_{v}=\cos \phi_{v} \sqrt{ }\left[a^{2}+b^{2}\right]+c
$$

If the two end points of the Small Circle path are at $A$ whose latitude is $\phi_{0}$ and longitude $\theta_{0}$ and $B$ whose latitude is $\phi_{n}$ and longitude $\theta_{n}$ then these points will have cartesian coordinates $\left(x_{0}, y_{0}, z_{0}\right)$ and $\left(x_{n}, y_{n}, z_{n}\right)$ and from equation (1) we find:

$$
\begin{aligned}
& z_{0}=a x_{0}+b y_{0}+c \\
& z_{n}=a x_{n}+b y_{n}+c
\end{aligned}
$$

The set of three equations (4), (5) and (6) form a simultaneous set from which we can determine the values of $a, b$ and $c$. In particular, the pair of equations (5) and (6) are a pair of linear equations in $a$ and $b$ so that we can solve these equations first to give us $a$ and $b$ in terms of $x_{0, n}, y_{0, n}, z_{0, n}$ and $c$. We find

$$
\begin{aligned}
& a=\frac{\left[y_{0} z_{n}-y_{n} z_{0}\right]+c\left[y_{n}-y_{0}\right]}{\left[x_{0} y_{n}-x_{n} y_{0}\right]} \\
& b=\frac{\left[x_{0} z_{n}-x_{n} z_{0}\right]+c\left[x_{n}-x_{0}\right]}{\left[x_{n} y_{0}-x_{0} y_{n}\right]}
\end{aligned}
$$

Substitution of these expressions for $a$ and $b$ into equation (4) will give us a quadratic equation of the form

$$
c^{2}+\alpha c+\beta=0
$$

where $\alpha$ and $\beta$ are numbers calculated from $x_{0, n}, y_{0, n}, z_{0, n}$ and $\phi_{v}$ in the following manner :

$$
\begin{aligned}
& \mu_{1}=\left[y_{0} z_{n}-y_{n} z_{0}\right] /\left[x_{0} y_{n}-x_{n} y_{0}\right] \\
& \mu_{2}=\left[y_{n}-y_{0}\right] /\left[x_{0} y_{n}-x_{n} y_{0}\right] \\
& \mu_{3}=\left[x_{0} z_{n}-x_{n} z_{0}\right] /\left[x_{n} y_{0}-x_{0} y_{n}\right] \\
& \mu_{4}=\left[x_{n}-x_{0}\right] /\left[x_{n} y_{0}-x_{0} y_{n}\right]
\end{aligned}
$$


then

$$
\begin{aligned}
& \alpha=-2\left[\sin \phi_{v}+\left(\mu_{1} \mu_{2}+\mu_{3} \mu_{4}\right) \cos ^{2} \phi_{v}\right] /\left[\mathrm{r}-\left(\mu_{2}^{2}+\mu_{4}^{2}\right) \cos ^{2} \phi_{v}\right] \\
& \beta=\left[\sin ^{2} \phi_{\mathrm{v}}-\left(\mu_{1}^{2}+\mu_{3}^{2}\right) \cos ^{2} \phi_{v}\right] /\left[\mathrm{I}-\left(\mu_{2}^{2}+\mu_{4}^{2}\right) \cos ^{2} \phi_{v}\right]
\end{aligned}
$$

This equation (9) is quadratic in $c$ and therefore has two solutions. This is because there are, in general, two arcs of Small Circles joining two points, $A$ and $B$, one of which takes the arc from $A$ to $B$, say, with difference of longitude $\left|\theta_{A}-\theta_{B}\right|$ and the other takes the $\operatorname{arc}$ from $A$ to $B$ in the opposite direction with difference of longitude $2 \pi-\left|\theta_{A}-\theta_{B}\right|$. The shorter arc is the one which gives the smaller absolute value of $c$. The equation can be solved quite easily to give $c$ whereby $a$ and $b$ can then be found from equations (7) and (8). We can then find $\theta_{v}$ from

$$
\tan \theta_{v}=\frac{b}{a}
$$

3. TO DETERMine THE RAdiUs OF THE SMALL CiRCle. Consider a figure in the plane of the meridian through the vertex of the Small Circle (Fig. 2). Let $N$ be the foot of the perpendicular from $O$, the centre of the sphere, to the plane containing the Small Circle. Let $O N=p$. $O N V$ is a right-angled triangle in the plane of the meridian. The length of the distance $O C$ along the $z$ axis is $c$.

The angle

$$
\operatorname{cov}=\frac{1}{2} \pi=\phi_{v}
$$

Applying the plane cosine formula to triangle $\mathrm{COV}$ we can determine the side $\mathrm{CV}$ and then, applying the sine formula we can determine angle $C V O(=\lambda$, say). The radius, $V N$, of the Small Circle circle $(=r$, say) is given by

$$
r=R \cos \lambda
$$

4. TO DETERMINE THE ANGLE SUBTENDED AT $N$ BY THE ARC $1 B$ AND ITS LENGTH. It can be deduced that the angle $N O C$ is equal to $\phi_{v}-\lambda$. The Cartesian coordinates, therefore, of the point $N$ are

where

$$
\begin{aligned}
x_{N} & =-p \sin \left(\phi_{v}-\lambda\right) \cos \theta_{v} \\
y_{N} & =-p \sin \left(\phi_{v}-\lambda\right) \sin \theta_{v} \\
z_{N} & =p \cos \left(\phi_{v}-\lambda\right) \\
P & =R \sin \lambda
\end{aligned}
$$

The vectors $N A\left(=\mathbf{r}_{A}\right)$ and $N B\left(=\mathbf{r}_{B}\right)$ are therefore given by

$$
\begin{aligned}
& \mathbf{r}_{A}=\left\{x_{0}+p \sin \left(\phi_{v}-\lambda\right) \cos \theta_{v}, y_{0}+p \sin \left(\phi_{v}-\lambda\right) \cos \theta_{v}, z_{0}-p \cos \left(\phi_{v}-\lambda\right)\right\} \\
& \mathbf{r}_{B}=\left\{x_{n}+p \sin \left(\phi_{v}-\lambda\right) \sin \theta_{v}, y_{n}+p \sin \left(\phi_{v}-\lambda\right) \sin \theta_{v}, z_{n}-p \cos \left(\phi_{v}-\lambda\right)\right\}
\end{aligned}
$$

If we let the angle $A N B$ between the two vectors be represented by $\gamma$ then, from the scalar product of the two vectors, we have

$$
\cos \gamma=\frac{\mathbf{r}_{A} \circ \mathbf{r}_{B}}{\left|\mathbf{r}_{A}\right| \cdot\left|\mathbf{r}_{B}\right|}
$$

Now $\left|\mathbf{r}_{A}\right|=\left|\mathbf{r}_{B}\right|=\mathbf{r}$ so that

$$
\cos \gamma=\frac{\mathbf{r}_{A} \circ \mathbf{r}_{B}}{r^{2}}
$$

and the length $s$ of the arc $A B$ is given by

$$
s=r \gamma \quad \text { where } \gamma \text { is measured in radians }
$$


5. EXAM PLE. Let us consider the route from a position off Punta del Este $\left(34^{\circ} 58^{\prime} \mathrm{S}\right.$, $\left.54^{\circ} 57^{\prime} \mathrm{W}\right)$ to a position off Fremantle $\left(32^{\circ} \circ 3^{\prime} \mathrm{S}, 115^{\circ} 45^{\prime} \mathrm{E}\right)$ without passing south of $60^{\circ} \mathrm{S}$

We have written a computer program to find the Small Circle track which has its vertex in the limiting latitude. We have computed its radius and the longitude of its vertex and we have found that

The Small Circle track will give a distance of 7 178.63 n.m.

The composite track will give a distance of $7147.60 \mathrm{n} . \mathrm{m}$.

The difference is $3 \mathrm{r} \times 3 \mathrm{n} . \mathrm{m}$.

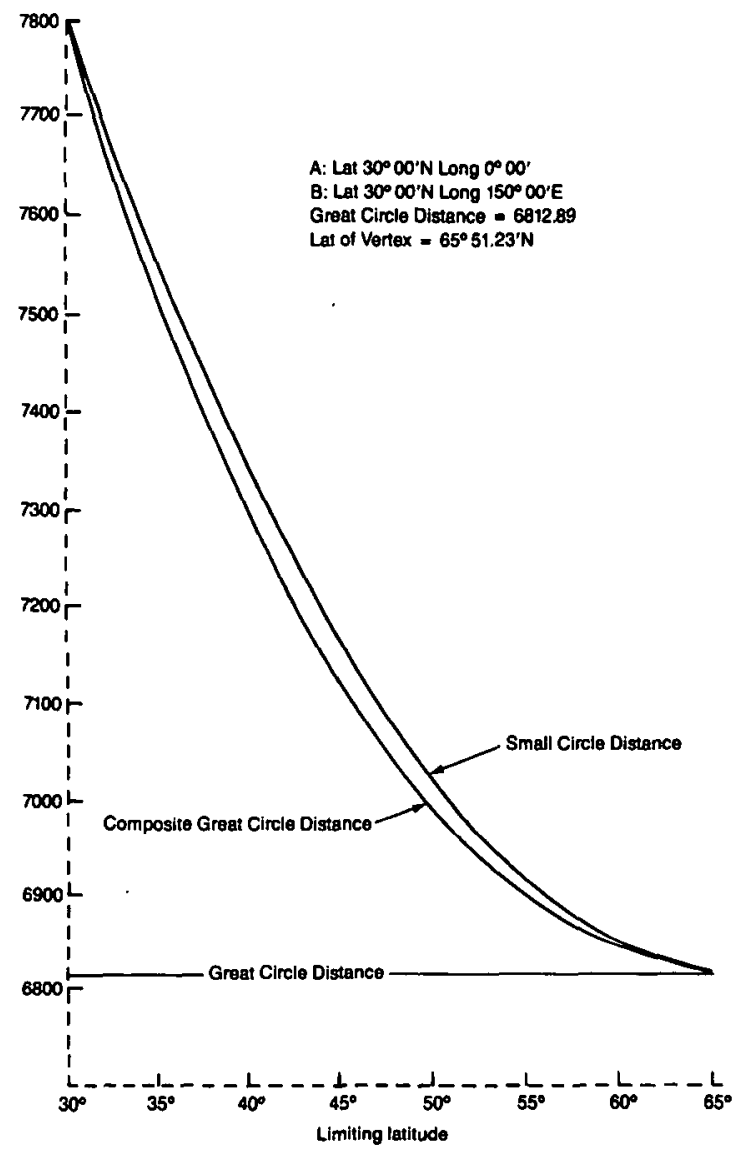

Fig. 3. Comparison of Small Circle and Composite Great Circle distances

The difference is really very small $(\approx 0.43$ per cent $)$ and the composite track will involve travelling $1101 \mathrm{n} . \mathrm{m}$. along the parallel of $60^{\circ} \mathrm{S}$. The distance along the full Great Circle track is 6744.96 n.m.

In section 6 we show some more detailed results of computations showing the comparison between the Composite Great Circle track and the Small Circle track and a graph (Fig. 3) highlighting the numerical results from section 6 (2). 
6. COMPARISON OF COMPOSITE GREAT CIRCLE AND SMALL CIRCLE ROUTES.

(1) A: Lat $20^{\circ} 00^{\prime} \mathrm{N}$ Long $0^{\circ} 00^{\prime} \mathrm{B}$ : Lat $20^{\circ} 00^{\prime} \mathrm{N}$ I $50^{\circ} 00^{\prime} \mathrm{E}$ Great Circle distance $=7822.29$ Lat of Vertex $=54^{\circ} 35^{\circ} 0^{\prime}$

\begin{tabular}{|c|c|c|c|c|}
\hline $\begin{array}{l}\text { Limiting } \\
\text { Latitude }\end{array}$ & $\begin{array}{c}\text { Composite } \\
\text { distance }\end{array}$ & $\begin{array}{c}\text { Small Circle } \\
\text { distance }\end{array}$ & Difference & $\%$ Diff \\
\hline $20^{\circ}$ & $8457 \cdot 23$ & $8457 \cdot 23$ & - & - \\
\hline $22.5^{\circ}$ & 8352.04 & $8372 \cdot 92$ & 20.88 & 0.25 \\
\hline $25^{\circ}$ & 826576 & 8293.77 & 28.01 & 0.34 \\
\hline $30^{\circ}$ & 8123.32 & 8151.98 & $28 \cdot 66$ & 0.35 \\
\hline $35^{\circ}$ & 801154 & 8033.69 & $22 \cdot 15$ & 0.28 \\
\hline $40^{\circ}$ & $7926 \cdot 60$ & 7940.08 & I 3.48 & 0.17 \\
\hline $45^{\circ}$ & $7867 \cdot 13$ & 7873.63 & $6 \cdot 50$ & 0.08 \\
\hline $50^{\circ}$ & 7832.51 & 7834.08 & $1 \cdot 57$ & 0.02 \\
\hline
\end{tabular}

(2) A : Lat $30^{\circ} 00^{\prime} \mathrm{N}$ Long $0^{\circ} 00^{\prime} \mathrm{B}$ : Lat $30^{\circ} 00^{\prime} \mathrm{N}$ Long $150^{\circ} 00^{\prime} \mathrm{E}$ Great Circle distance $=6812.89:$ Lat of Vertex $=65^{\circ} 51.23^{\prime}$

$\begin{array}{lllll}30^{\circ} & 7794.23 & 7794.23 & - & - \\ 32.5^{\circ} & 7636.63 & 7670.54 & 33.91 & 0.44 \\ 35^{\circ} & 7506.07 & 7553.61 & 47.54 & 0.63 \\ 40^{\circ} & 7289.90 & 7341.72 & 51.82 & 0.72 \\ 45^{\circ} & 7119.49 & 7161.82 & 42.83 & 0.59 \\ 50^{\circ} & 6988.60 & 7016.83 & 28.23 & 0.40 \\ 55^{\circ} & 6894.70 & 6909.28 & 14.58 & 0.21 \\ 60^{\circ} & 6836.55 & 6841.07 & 4.52 & 0.07\end{array}$

(3) A : Lat $40^{\circ} 00^{\prime} \mathrm{N}$ Long $0^{\circ} 00^{\prime} \mathrm{B}$ : Lat $40^{\circ} 00^{\prime} \mathrm{N}$ Long $150^{\circ} 00^{\prime} \mathrm{E}$ Great Circle distance $=5727^{\circ} 18:$ Lat of Vertex $=72^{\circ} 51^{\prime} 46^{\prime}$

$\begin{array}{lllll}40^{\circ} & 689440 & 6894.40 & - & - \\ 42.5^{\circ} & 6690.68 & 6735.61 & 44.93 & 0.67 \\ 45^{\circ} & 6522.91 & 6317.27 & 63.12 & 0.97 \\ 50^{\circ} & 6249.79 & 6317.27 & 67.48 & 1.08 \\ 55^{\circ} & 6041.10 & 6093.49 & 52.39 & 0.86 \\ 60^{\circ} & 5888.03 & 5919.59 & 31.56 & 0.54 \\ 65^{\circ} & 5786.69 & 5799.69 & 13.00 & 0.23 \\ 70^{\circ} & 5734.99 & 5736.81 & 1.82 & 0.03\end{array}$

7. CONCLUSION. It would seem that this alternative track - the track along the path of a Small Circle which reaches its vertex in the limiting latitude - might present an alternative to be considered by those concerned with weather routeing. In the examples that we have considered the difference in distance between the Small Circle track and the composite track is far less than the difference between the composite track and the full Great Circle track.

The position of points along the track of the Small Circle can be computed from equation (2). Given arbitrary values of the longitude; $\theta_{1}, \theta_{2}, \ldots, \theta_{n}$, we can determine the corresponding values of $\phi_{1}, \phi_{2}, \ldots, \phi_{n}$ and plot these points on the chart so that the track can be determined.

KEY WORDS
I. Great circles.
2. Small circles.
3. Voyage planning. 